\title{
Extraction of Solar Cell Modelling Parameters Using Differential Evolution Algorithm
}

\author{
Rituraj Tamrakar ${ }^{1}$, Archana Gupta ${ }^{2}$ \\ M.Tech Research Scholar, Dept. of Electronics and Telecommunication, Bhilai Institute of Technology, Durg, India ${ }^{1}$ \\ Professor, Department of Electrical Engineering, Bhilai Institute of Technology, Durg, India ${ }^{2}$
}

\begin{abstract}
Energy from the sun is the best option for electricity generation as it is abundantly available everywhere and sustainable source. Advance of Photovoltaic (PV) technology in recent years has made solar energy one of the practical alternative energy sources available in the energy market. In order to make the PV energy more affordable and cost effective, major focus of the research community and industry is improvement on power efficiency of PV systems. Parameter extraction of solar cell plays a crucial role in the simulation and design calculation of PV system. In this paper, in order to fast and accurately extract the solar cell parameters, differential evolution (DE) is proposed. The single diode and double diode model of solar cell is used as the basis for the extraction problem. The analysis carried out using current-voltage (I-V) characteristics showed that the proposed DE outperforms other methods, namely the genetic algorithm (GA) and partical swarm optimization (PSO).
\end{abstract}

Keywords: Solar cell models, parameter extraction, Differential evolution, Genetic algorithm, Particle swarm optimization.

\section{INTRODUCTION}

Renewable energy sources are getting added attention in recent years as alternative means of generating electricity in various parts of the world. Various motivations are promoting serious contribution of environmental friendly energy sources such as solar energy, wind energy, biomass, geothermal, hydro, wave energy etc in mass electricity production in many countries. Some of these reasons are: environmental concerns due to greenhouse effect, possible depletion and price increase of conventional energy primary resource. Solar energy is one of the most promising emission free resources that is currently being used worldwide to contribute to meeting rising demands of electric power with greater efficiency. Solar photovoltaic system is not only providing reliable and environmentally friendly energy but also could economically visible for rural and remote areas as an only source of energy. Despite the challenges of variability of electricity generation, life duration of solar photovoltaic devices and economic feasibility of deployment, it is gaining significant attention. This is due to the fact that solar photovoltaic is a semiconductor device that produces DC electricity when sunlight shines on the photovoltaic. It is static, quite, and free of moving parts system without any gas or toxic emissions and therefore requires little operation and maintenance costs [1].

India is a tropical country, where sunshine is available for longer hours per day and in great intensity. About 5,000 trillion $\mathrm{kWh}$ per year energy is incident over India's land area with most parts receiving $4-7 \mathrm{kWh}$ per sq. $\mathrm{m}$ per day. Solar energy, therefore, has great potential as future energy source. It also has the advantage of permitting the decentralized distribution of energy, thereby empowering people at the grassroots level. Solar also provides the ability to generate power on a distributed basis and enables rapid capacity addition with short lead times.
Off-grid decentralized and low-temperature applications will be advantageous from a rural electrification perspective. The National Solar Mission is a major initiative of the Government of India to promote ecologically sustainable growth while addressing India's energy security challenge. The solar mission targets to create an enabling policy framework for the deployment of $20,000 \mathrm{MW}$ of solar power by 2022 and a major research and development initiative to focus on improvement of efficiencies in existing materials, devices and applications and on reducing costs of balance of systems, establishing new applications by addressing issues related to integration and optimization [2].

For PV system, it is very important to select a model to closely resemble the characteristics of PV cells. Several models have been introduced to describe the currentvoltage (I-V) relationship in solar cells over recent years [3]. In practice, there are two main equivalent circuit models used to describe the non-linear I-V relationship: single and double diode models. However, no matter what kinds of models, there are important PV parameters that need to be accurately extracted for the simulation, design, performance evaluation, and control of solar cell systems. The main parameters that describe solar cell models behavior are the generated photocurrent, saturation current, series resistance, shunt resistance, and diode ideality factor. The electrical current produced by a solar cell depends on the intensity of the incident light and on its intrinsic properties. An accurate estimation of the cell parameters required for accurate performance evaluation [4].

The accuracy of PV module mainly depends on the accuracy of the PV module models and the extraction methods being used to determine the model's parameters. 
Several parameter extraction methods have been proposed in recent years which can be classified into three categories such as analytical, iterative and evolutionary computational methods.

Analytical methods [5-10] required less computational time for parameter extraction due to their simple structure and perform efficiently at standard testing conditions (STC) for some models but these methods are found to be unsuitable for solar model for wide range of changing weather conditions. Because, the mathematical model of $\mathrm{PV}$ panel represented is implicit in nature.

On the other hand, the iterative approaches [11-14] are based on mathematical algorithms and give more accurate results at STC but may fail in other weather conditions. Iterative methods based on newton raphson method (NRM) [15-17] were probably good option because of its accuracy and fast convergence, However NRM suffered from singularity problem. Also the convergence and accuracy were dependent on number of iterations.

Since the parameter extraction of solar cell models is a non-linear, multi-variable, and multi-modal problem with many local optima, traditional extraction techniques may be difficult to extract the parameters accurately. Therefore, in the later approaches, the use of evolutionary algorithms (EA) which uses artificial intelligence techniques for extraction of solar cell parameter has received considerable attention recently, such as genetic algorithms (GAs) [18, 19], particle swarm optimization (PSO) [20, $21,22]$, simulated annealing (SA) [23, 24], artificial bee swarm colony (ABC) [25, 26], Cuckoo search [27, 28] and so on. The evolutionary algorithms are population based global optimization algorithm which can be very effective regardless of gradient and initial condition information.

Differential evolution (DE), proposed by Storn and Price in 1997 [29], is a simple and efficient evolutionary algorithm for the numerical optimization. Due to several advantages like rapid convergence, ease of use, good accuracy, requires few control parameters, robust mutation schemes DE has been successfully used in diverse fields.

In this paper, the performance of DE for extraction of solar cell modeling parameters is investigated and compared to two other popular EA methods, GA and PSO. The rest of the paper is organized as follows, in section 2, the solar cell models used in this work together with the objective function to be optimized are introduced. Section 3 describes the DE algorithm proposed in this paper. The experimental results and analysis described in section 4 . Finally, Section 5 draws the conclusion of this paper.

\section{PROBLEM FORMULATION}

In the literature, many equivalent circuit models have been proposed to describe the I-V characteristics of solar cell. In practice, two main circuit models single diode model and double diode model are commonly used.

\section{A. Single Diode Model}

Due to simplicity and accuracy, the single diode model is considered. The single diode five parameter model, which include a current source, one diode and two resistors is shown in fig. 1

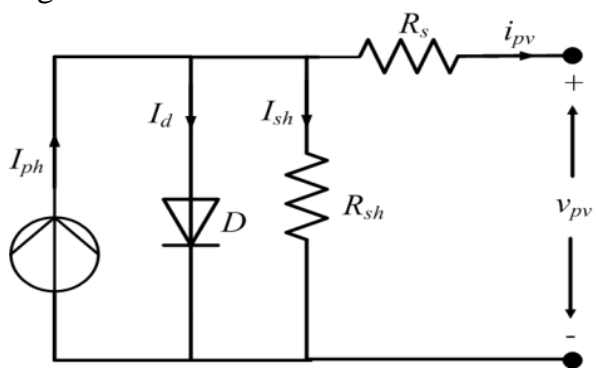

Fig. 1 - Single diode model of solar cell

The output current of the cell is calculated as follows:

$i_{p v}=I_{p h}-I_{0}\left[\exp \left(\frac{v_{p v}+i_{p v} R_{s}}{a V_{t}}\right)-1\right]-\left(\frac{v_{p v}+i_{p v} R_{s}}{R_{s h}}\right)$

Where $I_{p h}$ is cell generated photocurrent, $I_{0}$ is reverse saturation current of diode, $V_{t}(k \mathrm{~T} / q)$ is the thermal voltage of the solar cell, $k$ is the Boltzmann constant $\left(1.380650 \times 10^{-23} \mathrm{~J} / \mathrm{K}\right), \quad q$ is the electron charge $\left(1.602176 \times 10^{-19} \mathrm{C}\right)$, and $\mathrm{T}$ is the temperature of the $\mathrm{p}-\mathrm{n}$ junction in Kelvin. Variables $a$ is the diode ideality constants, $R_{s}$ and $R_{s h}$ are series resistance and shunt resistance respectively and $v_{p v}$ is output voltage of the cell. For the single diode model, five parameter to be extracted are parameters $I_{p h}, I_{0}, R_{s}, R_{s h}$, and $a$. An accurate knowledge of the parameters of solar cells is necessary for the design, control of solar cell and process optimization [14]

\section{B. Double Diode Model}

Another way to describe solar cell electrical behavior is to model it as current source connected in parallel with a rectifying diode. In practice the current source is also shunted by another diode that models the space charge recombination current and a shunt leakage resistor to account for the partial short circuit current path near the cell's edges due to the semiconductor impurities and nonidealities. Moreover, solar cell metal contacts and semiconductor material bulk resistance are represented by a resistor connected in series with the cell shunt elements [23]. The equivalent circuit for this model is shown in Fig. 2.

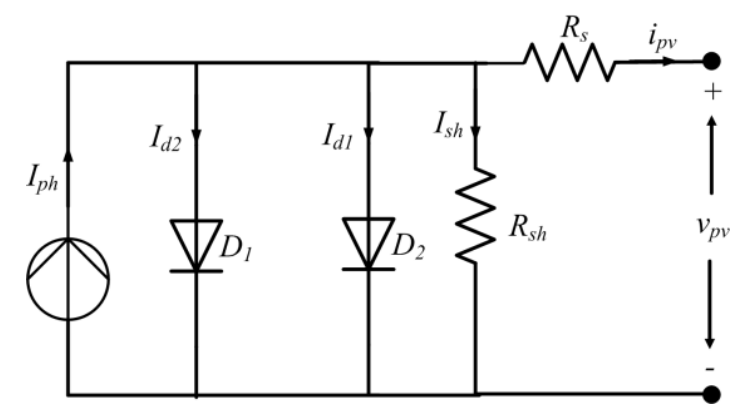

Fig. 2 - double diode model of solar cell

The output current of the solar cell can be formulated as:

$$
i_{p v}=I_{p h}-I_{d 1}-I_{d 2}-I_{s h}
$$

Where $I_{p h}$ is the cell generated photocurrent, $I_{d l}$ and $I_{d 2}$ are the first and second diode currents, respectively. $I_{s h}$ is 
shunt resistor current. The two diode currents $I_{d l}$ and $I_{d 2}$ few control parameters, rapid convergence and ease of and shunt current $I_{s h}$ can be calculated as,

$$
\begin{aligned}
I_{d 1} & =I_{01}\left[\exp \left(\frac{v_{p v}+i_{p v} R_{s}}{a_{1} V_{t}}\right)-1\right] \\
I_{d 2} & =I_{02}\left[\exp \left(\frac{v_{p v}+i_{p v} R_{s}}{a_{2} V_{t}}\right)-1\right] \\
I_{s h} & =\left(\frac{v_{p v}+i_{p v} R_{s}}{R_{s h}}\right)
\end{aligned}
$$

$I_{01}$ and $I_{02}$ are reverse saturation currents of diode1 and diode 2 respectively, $a_{1}$ and $a_{2}$ are the diode ideality constants. The complete physical behavior of double diode model of solar cell is in relation with seven parameters $I_{p h}$, $I_{01}, I_{02}, R_{s}, R_{s h}, a_{1}$ and $a_{2}$.

\section{Objective function}

It is noted that current equation is nonlinear transcendental function. In order to extract the parameters of different solar cell models from the I-V data using the optimization techniques, we first need to define the objective function to be optimized [4]. In this work the root mean square error is used as the objective function, which is described as

$$
\varepsilon=\sqrt{\frac{1}{N} \sum_{k=1}^{N} f_{k}\left(v_{p v}, i_{p v}, \emptyset\right)^{2}}
$$

Where $\mathrm{N}$ is the no. of experimental data, $\emptyset$ is decision vector which consists of the parameters to be extracted [3].

In case of single diode model the function $f\left(v_{p v}, i_{p v}, \emptyset\right)$ is given by

$$
\begin{gathered}
f\left(v_{p v}, i_{p v}, \emptyset\right)=I_{p h}-I_{0}\left[\exp \left(\frac{v_{p v}+i_{p v} R_{s}}{a V_{t}}\right)-1\right] \\
-\frac{v_{p v}+i_{p v} R_{s}}{R_{s h}}-i_{p v} \\
\varnothing=\left\{I_{p h}, I_{0}, R_{s}, R_{s h}, a\right\}
\end{gathered}
$$

For the double diode model, the function $f\left(v_{p v}, i_{p v}, \varnothing\right)$ is given by

$$
\begin{gathered}
f\left(v_{p v}, i_{p v}, \emptyset\right)=I_{p h}-I_{01}\left[\exp \left(\frac{v_{p v}+i_{p v} R_{s}}{a_{1} V_{t}}\right)-1\right] \\
-I_{02}\left[\exp \left(\frac{v_{p v}+i_{p v} R_{s}}{a_{2} V_{t}}\right)-1\right]-\frac{v_{p v}+i_{p v} R_{s}}{R_{s h}}-i_{p v}
\end{gathered}
$$

$\emptyset=\left\{I_{p h}, I_{01}, I_{02}, R_{s}, R_{s h}, a_{1}, a_{2}\right\}$

The aim of the experiment is to minimize Eq.6 with respect to $\varnothing$. The smaller the objective function, the better the solution obtained which implies the deviation between the module current and the computed current by the extraction method is small.

\section{DIFFERENTIAL EVOLUTION}

Differential evolution (DE) is a search and optimization algorithm which was proposed by storn and price in 1997 [29]. It is capable of handling non-differentiable, nonlinear, and multimodal objective functions with the advantages of ability to locate the accurate global optimum regardless of the initial parameter values, using

use. As an additional benefit DE is simple to understand and to implement and gives usable results in a reasonable amount of time has made DE one of the most popular and powerful tool in the field of optimization. DE was designed to be a stochastic direct search method which also has the advantage of being easily applied to experimental minimization where the cost function is derived from a physical experiment rather than a computer simulation.

DE algorithm is a population based direct search algorithm which initializes the population vector of fixed size randomly. This population is improved by applying mutation, crossover and selection operators. The process is repeated through the generations until the stopping criteria, predefined maximum iteration or a satisfactory fitness value is reached. At each generation the algorithm aims to create a new population by replacing points in the current population with better points. Through a repeated process the population is guided towards the global minimum.

The steps of the DE algorithm is given below:

\section{BEGIN Algorithm}

- Initialization $\}$

Create an initial population

- Evaluate each individual in the population

- Find out the vector with the lowest cost

- While the termination criterion not reached do

o Mutation

o Crossover

$\circ$ Evaluation and Selection

- $\{$ Return $\}$

$$
\text { Output } f * \text { and } \mathbf{x}^{*}
$$

END Algorithm

The operations are described as follows:

\section{A. Initialization}

The DE algorithm begins with a randomly initiated population $P^{G}$ of constant size NP consisting of Ddimensional real-valued parameter vectors $X_{i}^{G}, i=$ $1,2, \ldots, N P$ given by,

$$
P^{G}=\left[X_{1}^{G}, X_{2}^{G}, \ldots, X_{N P}^{G}\right], \quad G=1,2, \ldots G_{\max }
$$

Where $\mathrm{G}$ is the generation or iteration of the algorithm to which the population belongs, and $G_{\max }$ is the maximum number of generations defined by the user.

Each vector, also known as genome/chromosome, forms a candidate solution to the multi-dimensional optimization problem. Every parameter in the population is constrained to a certain search space and they cannot exceed either ends of the limits. The minimum and maximum bounds are denoted as $\left[X_{L}, X_{H}\right]$, where $X_{L}=\left[X_{1, L}, X_{2, L} \ldots, X_{D, L}\right]$ and $X_{H}=\left[X_{I, H}, \quad X_{2, H}, \ldots, X_{D, H}\right]$. Hence, the population is initialized randomly by assigning random values to each decision parameters of each individual of population and the $\mathrm{j}^{\text {th }}$ component of the $\mathrm{i}^{\text {th }}$ vector is initialized as,

$$
X_{i j}^{G}=X_{i j}^{L}+\operatorname{rand}(0,1) *\left(X_{i j}^{h}-X_{i j}^{L}\right)
$$


Where $\operatorname{rand}(0,1)$ is a uniformly distributed random value generated for each $\mathrm{j}$ and $X_{i j}^{h}$ and $X_{i j}^{L}$ are the respective upper and lower limits for the $\mathrm{j}^{\text {th }}$ variable or component. The population is improved by applying mutation, crossover and selection operators.

\section{B. Mutation}

The mutation operation is also known as differential mutation, which is the core operator of DE. A parent vector from the current generation is known as target vector $X_{i}^{G}$ and a mutant vector $V_{i}^{G}$ is generated by the mutation operation. Two vectors $X_{r 2}^{G}$ and $X_{r 3}^{G}$ are randomly selected from the population and the vector difference between them is established. This difference is multiplied by a scaling factor $F \in[0,1]$ and added to a third randomly chosen vector $X_{r 1}^{G}$ from the population. This is known as the differential variation and a mutant vector is generated as

$$
V_{i}^{G+1}=X_{r 1}^{G}+F *\left(X_{r 2}^{G}-X_{r 3}^{G}\right)
$$

\section{Crossover}

In order to diversify the current population, following mutation, DE employs the crossover operator to produce the trial vector $u_{i j}^{G+1}$ which is a combination of mutant vector $V_{i j}^{G+1}$ and a target vector $X_{i j}^{G}$. In DE two types of crossover methods are used, exponential (or two-point modulo) and binomial (or uniform). In exponential crossover, an integer $\mathrm{n}$ is randomly chosen among the numbers $[1, D]$. This integer behaves as a starting point in the target vector, from where the crossover or exchange of components with the mutant vector starts. Another integer $\mathrm{L}$ is chosen from the interval $[1, \mathrm{D}]$. $\mathrm{L}$ denotes the number of components the mutant vector actually contributes to the target vector. After choosing $\mathrm{n}$ and $\mathrm{L}$ the trial vector is obtained as,

$$
\begin{gathered}
u_{i j}^{G+1} \\
= \begin{cases}V_{i j}^{G+1} & \text { for } j=\langle\mathrm{n}\rangle_{\mathrm{D}},\langle\mathrm{n}+1\rangle_{\mathrm{D}}, \ldots,\langle\mathrm{n}+\mathrm{L}-1\rangle_{\mathrm{D}} \\
X_{i j}^{G} & \text { for all other } j \in[1, D]\end{cases}
\end{gathered}
$$

Where the angular brackets \langle\rangle$_{\mathrm{D}}$ represents a modulo function with modulus D.

In binomial crossover strategy, for each parameter, a random value is generated in the range of $[0,1]$ and is compared with user defined crossover constant CR. If the value of the random number is less than or equal to the value of the $\mathrm{CR}$, the parameter would be the mutant vector, otherwise the parameter would be the parent vector.

The binomial crossover strategy can be described as,

$$
= \begin{cases}u_{i j}^{G+1} & \text { if rand }[0,1] \leq C R \text { or } j=\operatorname{rnbr}(i) \\ X_{i j}^{G} & \text { otherwise }\end{cases}
$$

Where $\operatorname{rnbr}(\mathrm{i})$ is randomly chosen index $\in 1,2, \ldots, \mathrm{D}$ which insures that $u_{i j}^{G+1}$ gets at least one parameter from $V_{i j}^{G+1}$

\section{Constraint Handling}

In boundary constrained problem it is essential to ensure that parameter values lie inside their allowed ranges. A simple way to replace parameter values that violate boundary constraints with random values generated within the feasible range is as follows [30].

$$
u_{i j}^{(G+1)}=\left\{\begin{array}{lr}
x_{i j}^{(U)}+\operatorname{rand}[0,1] *\left(x_{i j}^{(G)}-x_{i j}^{(U)}\right) & \text { if } u_{i j}^{(G+1)}>x_{i j}^{(U)} \\
x_{i j}^{(L)}+\operatorname{rand}[0,1] *\left(x_{i j}^{(G)}-x_{i j}^{(L)}\right) & \text { if } u_{i j}^{(G+1)}>x_{i j}^{(L)} \\
u_{i j}^{(G+1)} & \text { otherwise }
\end{array}\right.
$$

\section{E. Evaluation and selection}

Finally, to keep the population size constant in the following generations, the selection operation is employed to determine whether the trial or the target vector survives to the next generations. To decide whether or not it should become a member of generation $\mathrm{G}+1$, the trial vector $u_{i}^{G+1}$ is compared to the target vector $X_{i}^{G}$ using the greedy criterion. If vector $u_{i}^{G+1}$ yields a smaller cost function value than $X_{i}^{G}$, then $X_{i}^{G+1}$ is set to $u_{i}^{G+1}$; otherwise, the old value is $X_{i}^{G}$ retained.

$X_{i}^{G+1}= \begin{cases}u_{i}^{G+1} & \text { if } f\left(u_{i}^{G+1}\right) \leq f\left(X_{i}^{G}\right) \\ X_{i}^{G} & \text { otherwise }\end{cases}$

Where $f\left(X_{i}^{G}\right)$ is objective function to be optimized.

\section{EXPERIMENTAL RESULT AND ANALYSIS}

The performance of DE is evaluated for extraction of single diode and double diode model of solar cell and compared with those of GA and PSO. The extracted parameters obtained are selected from the best of ten independent runs of each algorithm. The experimental I-V data of solar cell is obtained from [31]. In this work, the population size NP $=50$ is used for all the three methods. The maximum iteration is set to 10,000 . The mutation factor $(F)$ is set at 0.95 the crossover rate is chosen to be 0.8 . DE/current-to-best/1/bin strategy is employed for the DE. The search range for single and double diode models were set as follows:

$$
\begin{gathered}
I_{p h} \in[0,1], I_{01,2} \in[0,1 . e-6], R_{s} \in[0,0.5], \\
R_{s h} \in[0,100], a_{1,2} \in[1,2]
\end{gathered}
$$

For single diode model, Table 1 shows extracted parameters and RMSE value of DE are compared with GA and PSO. It is clear to observe that DE provides best RMSE value among GA and PSO.

Table 1: Extracted parameters using different methods for single diode model.

\begin{tabular}{|c|c|c|c|}
\hline parameters & DE & GA & PSO \\
\hline$I_{p h}(A)$ & 0.7608 & 0.7604 & 0.7684 \\
\hline$I_{0}(\mu A)$ & 0.3230 & 0.8132 & 0.2664 \\
\hline$R_{S}(\Omega)$ & 0.0364 & 0.03240 & 0.0375 \\
\hline$R_{s h}(\Omega)$ & 53.7185 & 82.0064 & 100.00 \\
\hline$a$ & 1.3956 & 1.4890 & 1.3770 \\
\hline RMSE & $9.8602 \mathrm{E}-04$ & 0.0020 & 0.0015 \\
\hline
\end{tabular}


For double diode model, seven parameters need to be extracted. The results of DE are compared with GA and PSO in table 2.

Table 2: Extracted parameters using different methods for double diode model.

\begin{tabular}{|c|c|c|c|}
\hline Parameters & DE & GA & PSO \\
\hline$I_{p h}(A)$ & 0.7608 & 0.7605 & 0.7621 \\
\hline$I_{01}(\mu A)$ & 0.2398 & 0.9106 & 1.0000 \\
\hline$I_{02}(\mu A)$ & 1.0000 & 0.1106 & 0.9899 \\
\hline$R_{S}(\Omega)$ & 0.0367 & 0.0318 & 0.0310 \\
\hline$R_{S h}(\Omega)$ & 55.6025 & 99.6095 & 100.00 \\
\hline$a_{1}$ & 1.3712 & 1.5021 & 2.0000 \\
\hline$a_{2}$ & 2.0000 & 1.1667 & 1.5160 \\
\hline RMSE & $9.8113 \mathrm{E}-04$ & 0.0023 & 0.0017 \\
\hline
\end{tabular}

\section{CONCLUSION}

In this paper, the proposed DE was applied to different models of solar cells and their parameters, generated photocurrent, saturation current, series resistance, shunt resistance, and diode ideality factor had been extracted. DE had comprehensively evaluated through the parameter extraction problems of different solar cell models, i.e. single diode and double diode model. The performance of DE was compared with GA and PSO. Experimental results confirmed that the proposed DE method was able to extract the parameters of different solar cell models accurately. DE can provide more precise and robust solution, obtain higher success rate, and converge faster.

\section{REFERENCES}

1] K. M. El-Naggar, M. R. AlRashidi, M. F. AlHajri and A. K. Al-Othman, "Simulated Annealing algorithm for photovoltaic parameters identification," Solar Energy, vol. 86, no.1, pp. 266-274, 2012.

2] Ministry of new and renewable energy, annual report (2014-2015). Jawaharlal Nehru National Solar Mission towards Building solar India, Government of India. Available: http://www.mnre.gov.in/filemanager/UserFiles/mission_document_JNN SM.pdf.

[3] M. Wolf, G. Noel and R. Stirn, "Investigation of the double exponential in the current-voltage characteristics of silicon solar cells," IEEE Transactions on Electron Devices, vol. 24, no. 4, pp. 419 - 428, April 1977

[4] M. F. AlHajri, K. M. El-Naggar, M. R. AlRashidi and A. K. Al-Othman, "Optimal extraction of solar cell parameters using pattern search," Renewable Energy, vol. 44, pp. 238-245, 2012.

[5] M. R. AlRashidi, K. M. El-Naggar and M. F. AlHajri, "Heuristic Approach for Estimating the Solar Cell Parameters," Recent Researches in Applied Information Science, pp. 80-83, Published by WSEAS Press, 2012

[6] M. de Blas, J. Torres, E. Prieto and A. Gracia, "Selecting a suitable model for characterizing photovoltaic devices," Renewable Energy, vol. 25 , no. 3, pp. 371-380, 2002.

[7] A. Jain, N. S. Singh and A. Kapoor, "An Exact Analytical Method for Calculating the Parameters of a Real Solar Cell Using Special Trans Function Theory(STFT)," International Journal of Renewable Energy, vol.3, no.1, pp. 201-206, 2012.

[8] C. Zhang, J. Zhang, Y. Hao, Z. Lin and C. Zhu, 'A simple and efficient solar cell parameter extraction method from a single current-voltage curve," Journal of applied physics, vol. 110, no. 6, 2011

[9] A. Laudani, F. Riganti Fulginei and A. Salvini, "High performing extraction procedure for the one-diode model of a photovoltaic panel from experimental I-V curves by using reduced forms," Solar Energy, vol. 103, pp. 316-326, 2014

[10] J. Ma, K. L. Man, T. O. Ting, N. Zhang, E. G. Lim, S. Guan, P. W. H. Wong, T. Krilavicius, D. Saulevicius and C. U. Lei, "Simple Computational Method of Predicting Electrical Characteristics in Solar
Cells," Electronics and Electrical Engineering, vol. 20, no. 1, pp. 41-44, 2014

[11] R Khezzar, M. Zereg, and A. Khezzar, "Comparative Study of Mathematical Methods for Parameters Calculation of Current-Voltage Characteristic of Photovoltaic Module," IEEE international conference on Electrical and Electronics Engineering (ELECO), Nov. 2009, pp. 2428

[12] A. Mohapatra, B. K. Nayak and K. B. Mohanty, "Comparative study on single diode photovoltaic module parameter extraction methods," IEEE International Conference on Power, Energy and Control (ICPEC), Feb. 2013, pp. 30-34.

[13] F. Ghani, M. Duke and J. Carson, "Extraction of solar cell modeling parameters using the Lambert W function," in Australian Solar Energy Society Melbourne, Dec. 2012.

[14] B. Subudhi and R. Pradhan, "Development of new parameter extraction scheme and maximum power point tracking system," PHD thesis, National Institute of Technology, Rourkela, July 2014.

[15] K. Ishaque, Z. Salam and H. Taheri, "Accurate MATLAB Simulink PV System Simulator Based on a Two-Diode Model," Journal of Power Electronics, vol. 11, no. 2, pp. 179-187, 2011.

[16] M. Chegaar, Z. Ouennoughi, F. Guechi and H. Langueur, "Determination of Solar Cells Parameters under Illuminated Conditions," Journal of Electron Devices, vol. 2, pp. 17-21, 2003.

[17] N. Enebish, D. Agchbayar, S. Dorjkhand, D. Baatar and I. Ylemj, "Numerical analysis of solar cell current-voltage characteristics," Solar energy materials and solar cells, vol. 29, no. 3, pp. 201-208, 1993.

[18] M. Zagrouba, A. Sellami, M. Bouaicha and M. Ksouri, "Identification of PV solar cells and modules parameters using the genetic algorithms: application to maximum power extraction," Solar energy, vol. 84 , no. 5, pp. 860-866, 2010 .

[19] N. Moldovan, R. Picos and E. G. Moreno, "Parameter extraction of a solar cell compact model using genetic algorithms," IEEE proceedings of the 2009 Spanish Conference of Electron Devices, Feb 2009, pp. 379 382

[20] C. Saravanan and M. A. Panneerselvam, "A Comprehensive Analysis for Extracting Single Diode PV Model Parameters by Hybrid GA-PSO Algorithm," International Journal of Computer Applications, vol. 78, no.8, pp. 16-19, September 2013.

[21] H. Qin and J. W. Kimball, "Parameter Determination of Photovoltaic Cells from Field Testing Data using Particle Swarm Optimization," IEEE Power and Energy Conference at Illinois , 2011, pp. 1-4

[22] M. Ye, X. Wang and Y. Xu, "Parameter extraction of solar cells using particle swarm optimization," Journal of Applied Physics, vol. 105, no. 9, May 2009.

[23] M. R. AlRashidi, K. M. El-Naggar and M. F. AlHajri, "Solar Cell Parameters Estimation Using Simulated Annealing Algorithm," world academy of science, engineering and technology, vol. 7, no. 4, pp. 149152,2013

[24] M. R. AlRashidi, K. M. El-Naggar and M. F. AlHajri, "Extraction of Photovoltaic Characteristics Using Simulated Annealing," International Conference on Advances in Engineering Sciences and Applied Mathematics, May2014.

[25] M. Ketkar and M. Chopde, "Efficient Parameter Extraction of Solar Cell using Modified ABC," International Journal of Computer Applications, vol. 102 , no. 1 , Sept. 2014.

[26] D. Oliva, E. Cuevas and G. Pajares, "Parameter identification of solarcells using artificial bee colony optimization," Energy, vol. 72, pp. 93-102, 2014.

[27] J. Ma, T. O. Ting, K. L. Man, N. Zhang, S. U. Guan and P. W. Wong, "Parameter estimation of photovoltaic models via cuckoo search," Journal of Applied Mathematics, vol. 2013, 8 pages, 2013.

[28] R. Jovanovic, S. Kais and F. H. Alharbi, "Cuckoo Search Inspired Hybridization of the Nelder-Mead Simplex Algorithm Applied to Optimization of Photovoltaic Cells," arXiv preprint, nov. 2014.

[29] R. Storn and K. Price, "Differential Evolution, A Simple and Efficient Heuristic Strategy for Global Optimization over Continuous Spaces," Journal of Global Optimization, vol. 11, pp. 341-359, 1997.

[30] J. Lampinen, "Global Optimization by Differential Evolution. Technical Report, Lappeenranta University of Technology, Lappeenranta, Finland, 1999.

[31] T. Easwarakhanthan, J. Bottin, I. Bouhouch, C. Boutrit, "Nonlinear minimization algorithm for determining the solar cell parameters with microcomputers." International Journal of Solar Energy, vol. 4, no. 1, pp. 1-12, 1986 